\title{
Rapid identification of bacteria and candida using pna-fish from blood and peritoneal fluid cultures: a retrospective clinical study
}

Dana $\mathrm{M} \mathrm{Harris}^{1}$ and D Jane Hata ${ }^{2^{*}}$

\begin{abstract}
Background: Peptide nucleic acid fluorescent in situ hybridization (PNA-FISH) is a rapid and established method for identification of Candida sp., Gram positive, and Gram negative bacteria from positive blood cultures. This study reports clinical experience in the evaluation of 103 positive blood cultures and 17 positive peritoneal fluid cultures from 120 patients using PNA-FISH. Our study provides evidence as to potential pharmaceutical cost savings based on rapid pathogen identification, in addition to the novel application of PNA-FISH to peritoneal fluid specimens.
\end{abstract}

Methods: Identification accuracy and elapsed time to identification of Gram positives, Gram negatives, and Candida sp., isolated from blood and peritoneal fluid cultures were assessed using PNA-FISH (AdvanDx), as compared to standard culture methods. Patient charts were reviewed to extrapolate potential pharmaceutical cost savings due to adjustment of antimicrobial or antifungal therapy, based on identification by PNA-FISH.

Results: In blood cultures, time to identification by standard culture methods for bacteria and Candida sp., averaged 83.6 hours ( $95 \% \mathrm{Cl} 56.7$ to 110.5). Identification by PNA-FISH averaged 11.2 hours ( $95 \% \mathrm{Cl} 4.8$ to 17.6). Overall PNA-FISH identification accuracy was $98.8 \%$ (83/84, 95\% Cl 93.5\% to 99.9\%) as compared to culture. In peritoneal fluid, identification of bacteria by culture averaged 87.4 hours $(95 \% \mathrm{Cl}-92.4$ to 267.1). Identification by PNA-FISH averaged 16.4 hours (95\% Cl -57.3 to 90.0). Overall PNA-FISH identification accuracy was 100\% (13/13, 95\% Cl 75.3\% to 100\%). For Candida sp., pharmaceutical cost savings based on PNA-FISH identification could be $\$ 377.74 /$ day. For coagulase-negative staphylococcus (CoNS), discontinuation of vancomycin could result in savings of $\$ 20.00 /$ day.

Conclusions: In this retrospective study, excellent accuracy of PNA-FISH in blood and peritoneal fluids with reduced time to identification was observed, as compared to conventional culture-based techniques. Species-level identification based on PNA-FISH could contribute to notable cost savings due to adjustments in empiric antimicrobial or antifungal therapy as appropriate to the pathogen identified.

Keywords: PNA-FISH, Blood culture, Bacteremia, Fungemia, Peritoneal fluid

\section{Background}

Rapid identification of pathogens in blood and peritoneal fluid plays a critical role in patient outcomes, and healthcare cost. Bloodstream associated infections (BSI) are the $10^{\text {th }}$ leading cause of death in the U.S [1]; this rate has increased by $78 \%$ over the last 2 decades. Bloodstream infections acquired in hospitals account for approximately

\footnotetext{
* Correspondence: hata.donna@mayo.edu

${ }^{2}$ Department of Laboratory Medicine and Pathology, Mayo Clinic College of Medicine, 4500 San Pablo Rd, Jacksonville, Florida 32224, USA

Full list of author information is available at the end of the article
}

250,000 cases each year with a direct attributable mortality rate of $16-40 \%$. Total hospitalization time is prolonged by 4.5-32 days [2], and inappropriate empirical antimicrobial therapy is an important predictor of death in this patient population [3].

In identification of organisms implicated in BSI, automated blood culture followed by biochemical analysis of isolates is considered the gold standard [4]. New detection technologies for organism identification from blood culture such as real-time PCR, DNA microarrays, matrix-assisted laser desorption ionization time of flight 
mass spectrometry (MALDI-TOF), and peptide nucleic acid fluorescent in situ hybridization (PNA-FISH) for detection of Candida, Gram positive, and Gram negative organisms may increase organism identification accuracy, and significantly reduce time to result [5].

PNA-FISH is a U.S. Food and Drug Administration (FDA) approved commercially available method for the detection of bacteria and yeast species directly from positive blood culture bottles. This methodology utilizes hybridization of PNA probes to organism-specific rRNA, with detection via fluorescent microscopy [6,7]. To date, studies utilizing PNA-FISH have been reported for detection of Gram positive organisms, Candida, and other Gram negative species [3,8-12]. Although not specifically FDA-approved for this indication, our study also assessed identification accuracy from peritoneal fluid inoculated into blood culture media. We are unaware of other published studies evaluating PNA-FISH for identifying organisms from peritoneal fluid.

The purpose of our study was to compare PNA-FISH to traditional culture techniques in blood and peritoneal fluids for accuracy in identification to species, and overall time to identification of Gram positive organisms, Gram negative organisms and Candida spp. In a secondary analysis, we specifically examined the potential pharmaceutical cost savings when the correct species of Candida was identified with concomitant correction of antifungal therapy as necessary. This manner of analysis was also applied to vancomycin costs when identification of coagulase-negative Staphylococcus (CoNS) was achieved by PNA-FISH in blood cultures.

\section{Methods}

This study was approved by the Mayo Clinic Institutional Review Board. This study retrospectively evaluated blood or peritoneal fluid cultures from 120 patients routinely submitted to the Clinical Microbiology Laboratory at Mayo Clinic in Jacksonville, FL from February through June 2009. Testing was performed using both traditional blood culture methodology and PNA-FISH (AdvanDx, Woburn, MA). PNA-FISH testing and standard blood culture workup was performed by two different technologists, with reciprocal blinding of results.

\section{Blood/peritoneal fluid cultures}

Blood cultures and peritoneal fluid cultures were performed using BACTEC Plus Aerobic F and Plus Anaerobic F bottles (Becton Dickinson, Sparks, MD) using standard methods and according to the manufacturer's recommendations [4]. As part of a routine culture set consisting of one aerobic and one anaerobic bottle, 8.0 to $10.0 \mathrm{~mL}$ of blood or peritoneal fluid were inoculated in each BACTEC bottle and incubated at $37^{\circ} \mathrm{C}$. When the first bottle of the pair signaled positive by the BACTEC 9240 instrument and confirmed by Gram stain, an aliquot from the bottle was subcultured to a blood agar plate and incubated at $37^{\circ} \mathrm{C}$. After sufficient pure bacterial growth was achieved ( $24-88$ hours), identification tests were performed on a Microscan Walkaway-96 system utilizing a 24 hour incubation period using GP-33 or GN-34 combination panels as appropriate (Siemens Healthcare Diagnostics, Deerfield IL).

\section{PNA-FISH}

Blood culture bottles signaling positive in the BACTEC system were tested on the same day with PNA-FISH. Based on Gram stain results from the signal-positive blood culture bottle, a specific PNA-FISH probe was selected for use. These consisted of the S. aureus/CoNS, E. faecalis/OE (other Enterococci, E. faecium), E. coli/P. aeruginosa, EK (E. coli, K. pneumoniae/P. aeruginosa). For yeasts, probes for C. albicans/C. glabrata, and the Yeast Traffic Light (C. albicans/C. parapsilosis - green, C. glabrata/C. krusei - red, C. tropicalis - yellow) were utilized. The PNA-FISH method was performed according to manufacturer's recommendations. One drop of fixation solution was gently mixed with one drop $(10 \mu \mathrm{l})$ of specimen from a positive BACTEC bottle on a PNAFISH slide. Slides were fixed in methanol, followed by immersion in $80 \%$ ethanol for 10 minutes. Slides were allowed to air dry. One drop of specific PNA probe mixture was applied to the slide, a cover slip was applied, and the slide was hybridized for 90 minutes at $55^{\circ} \mathrm{C}$. Post-hybridization, slides were incubated in $55^{\circ} \mathrm{C}$ wash solution for 30 minutes. Slides were allowed to air dry, and mounted. Slides were examined at $60 \mathrm{X}$ on an Olympus BX-41 fluorescent microscope equipped with a 528-633 dual band filter. Slides were examined for the presence of multiple bright fluorescent (green, red, yellow), morphologically consistent microorganisms in multiple fields of view. Organisms resembling yeast were initially tested with the C. albicans/C. glabrata PNA-FISH probe. If negative, PNA-FISH was repeated with the Yeast Traffic Light probe in order to identify presence of C. parapsilosis, C. krusei, or C. tropicalis. Gram-negative organisms were initially tested with the E. coli/P. aeruginosa probe. If negative, PNA-FISH was repeated with the EK/P. aeruginosa probe in order to identify presence of Klebsiella pneumoniae.

Probe-specific PNA-FISH positive and negative control slides were obtained from the manufacturer and utilized for quality control each time PNA-FISH was performed. All bacterial and yeast identifications from blood and peritoneal fluid cultures were confirmed using standard methods by the Clinical Microbiology Laboratory at Mayo Clinic in Jacksonville, FL.

Identification accuracy and time to identification using both PNA-FISH and culture (gold standard) were 
assessed. Time to identification with culture (C-ID) was calculated from the time the BACTEC bottle signaled positive, to the time at which the microbiology laboratory identified the organism. Time to identification with PNA-FISH (F-ID) was calculated from the time the BACTEC bottle initially signaled positive, to completion of F-ID. Appropriate controls were performed with each PNA-FISH test batch. PNA-FISH was performed in batches twice daily at $8 \mathrm{am}$ and $1 \mathrm{pm}$. All samples that became BACTEC positive between those times were held until the next processing period, and the time elapsed until processing was added to the total F-ID time. Time saved was defined as the difference between C-ID and F-ID. Organisms that could not be identified by PNAFISH were not included in C-ID or F-ID calculations.

In order to better assess detection and identification of Candida spp., 15 BACTEC bottles were spiked (9 blood, 6 peritoneal fluid) by mixing $8.0 \mathrm{~mL}$ of culture-negative blood or peritoneal fluid with $2.0 \mathrm{~mL}$ of a 0.5 McFarland suspension of a known species of yeast and inoculating

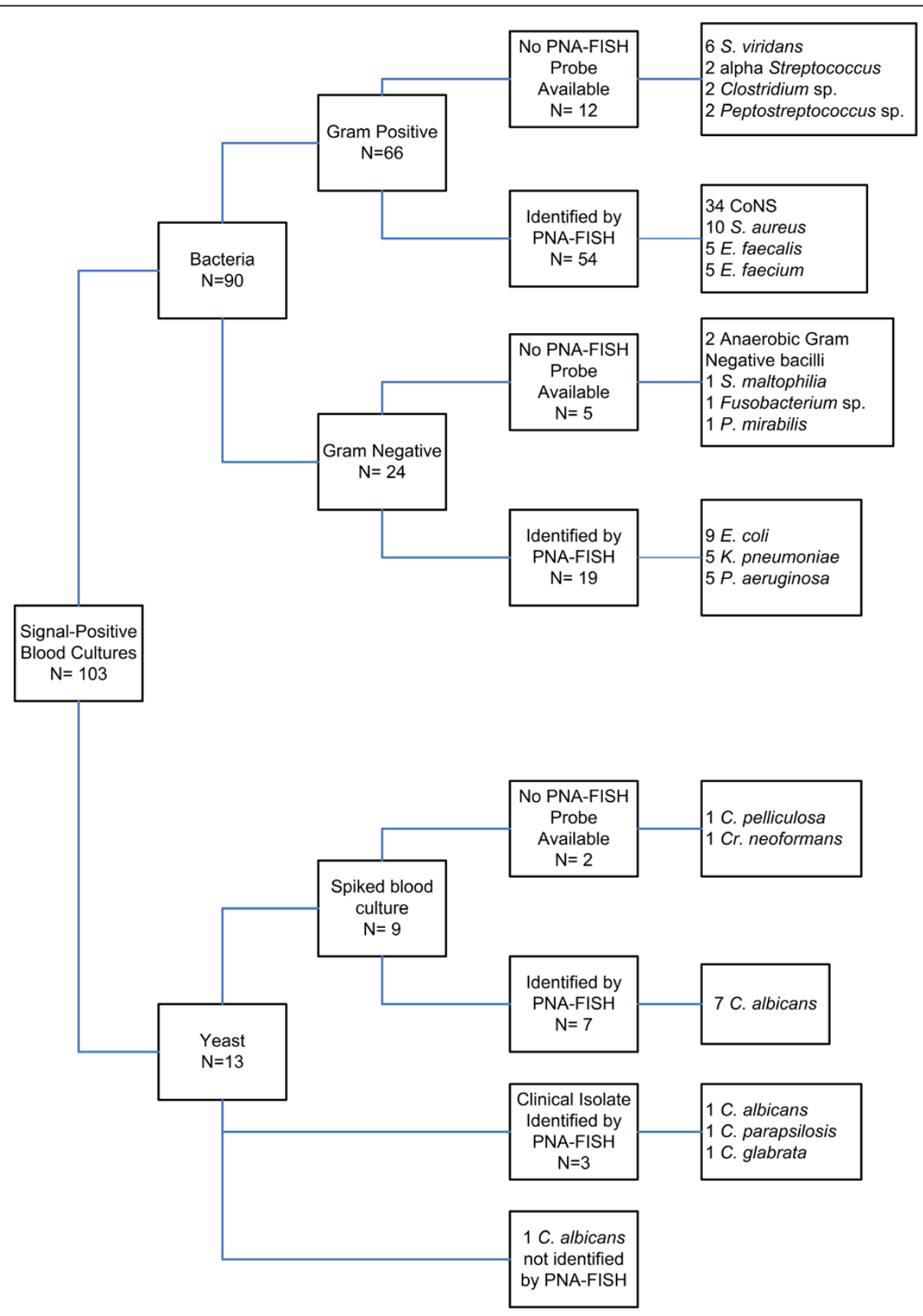

Figure 1 Analysis of Blood Culture by PNA-FISH. 
the BACTEC bottle. Bottles were incubated under standard conditions until they signaled positive. At that time the bottle was removed from the BACTEC instrument and processed as previously described. The results from these spiked bottles were included only in the accuracy calculations, and not calculated time to detection. Statistical significance of time to C-ID versus time to F-ID was calculated using $\mathrm{t}$ test method and GraphPad [13].

Patient charts were reviewed for selection of empiric antimicrobial in order to extrapolate potential pharmaceutical cost savings of PNA-FISH. Pharmaceutical costs were based on the 2009 institutional Average Wholesale Price (AWP). The AWP for oral fluconazole $400 \mathrm{mg}$ daily was $\$ 27.26$, and caspofungin $50 \mathrm{mg}$ daily was $\$ 405.00$. The AWP of IV vancomycin 2 grams daily was $\$ 20.00$ [14].

\section{Results}

A total of 103 blood culture bottles and 17 peritoneal fluid culture bottles were signal-positive for bacterial or fungal growth after incubation in the BACTEC system. Of those, 9 blood and 6 peritoneal fluid culture bottles had been spiked with Candida sp. or other yeasts in the laboratory in order to increase sample numbers to statistically meaningful levels specific for the pathogen. Compared to traditional culture methods, 96 of 120 positive BACTEC blood culture bottles contained an organism for which a PNA-FISH probe was available. Twentythree signal-positive bottles contained a bacteria or yeast for which a PNA-FISH probe was not available [Figure 1, Figure 2]. All 23 of these bottles tested negative by PNA-FISH probes used in this study.

In signal-positive blood culture bottles containing Gram positive bacteria, the following species were identified by PNA-FISH: 34 coagulase-negative Staphylococcus [CoNS], $10 \mathrm{~S}$. aureus, $5 \mathrm{E}$. faecalis, $5 \mathrm{E}$. faecium. In signal-positive blood culture bottles containing Gram negative bacteria, the following species were identified: 9 E. coli, $5 \mathrm{~K}$. pneumoniae, 5 P. aeruginosa. In signal positive blood culture bottles containing yeasts, the following species were identified: 8 C. albicans, 1 C. glabrata, 1 C. parapsilosis. One isolate from a positive blood culture bottle confirmed as $C$. albicans reacted only weakly with the C. albicans/C. glabrata dual probe as well as the PNA-FISH Traffic Light, and was judged to be negative by PNA-FISH. Of the 9 blood culture bottles spiked with yeasts; 7 positive bottles containing C. albicans were correctly identified by PNA-FISH. Two spiked bottles contained a yeast for which a PNA-FISH probe was not available (C. pelliculosa, Cr. neoformans); both bottles were negative with the yeast dual probe and Traffic Light probe [Figure 1]. Spiked bottles were included for accuracy calculations, but not included in calculation of time to results.

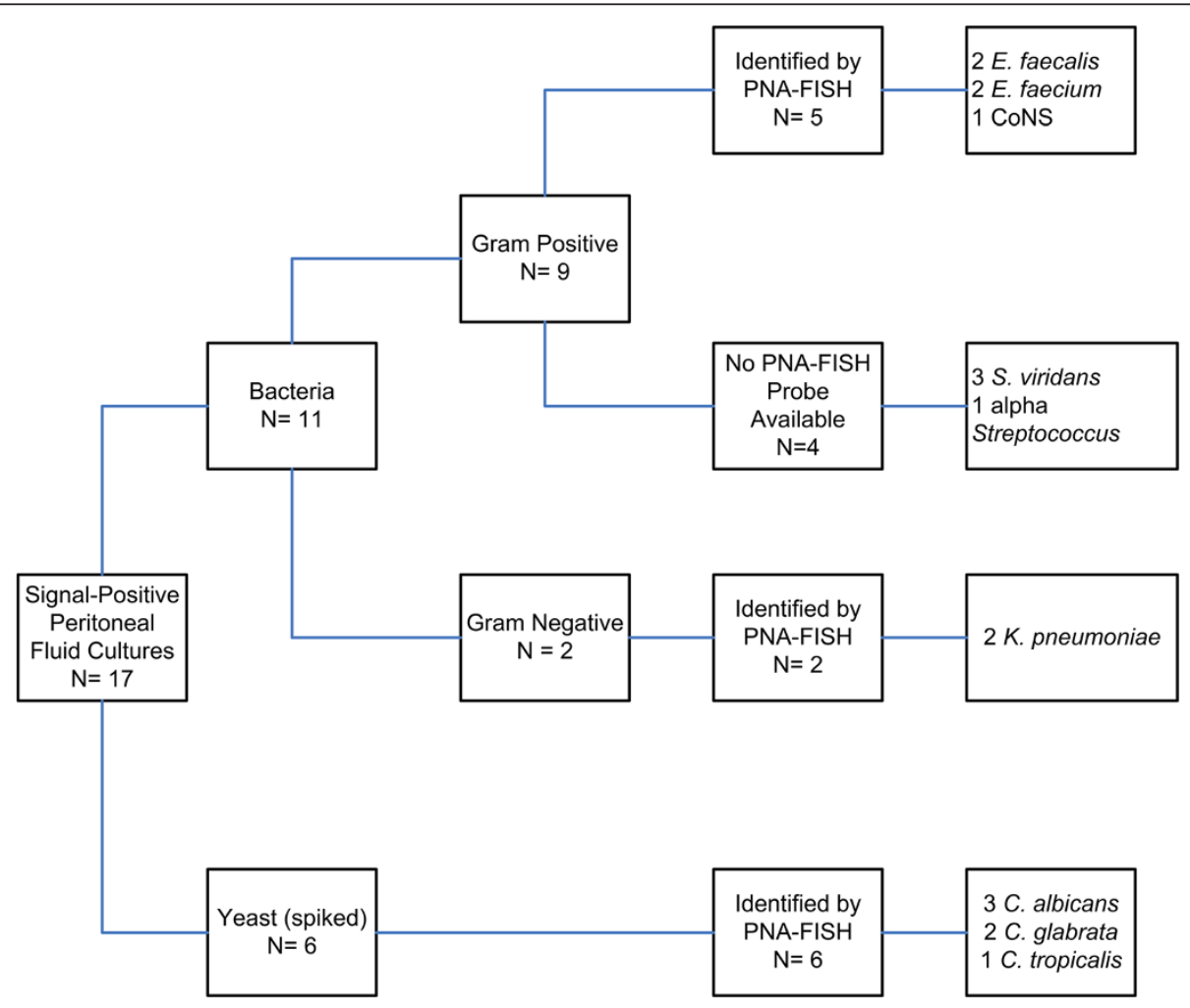

Figure 2 Analysis of Peritoneal Fluid Cultures by PNA-FISH. 
Compared to conventional blood culture identification, Gram positive organisms were identified 70.2 hours faster with PNA-FISH (95\% CI 60.2 to 80.3, p< 0.0001) and Gram negative organisms were identified 64.1 hours more rapidly (95\% CI 51.0 to $77.2, \mathrm{p}=0.0002)$. Candida sp. were identified 83.0 hours faster with PNA-FISH compared to culture (95\% CI 33.8 to $132.3 \mathrm{p}=0.0095)$ [Table 1]. For bacteria, overall accuracy of identification with PNA-FISH compared to culture was $100 \%$ ( $73 / 73$, 95\% CI $95 \%$ to $100 \%)$. Overall accuracy of Candida sp. identification was $90.9 \%$ (10/11, $95 \%$ CI $58.7 \%$ to $99 \%)$.

Results from peritoneal fluid cultures were analyzed separately from blood cultures due to potential differences in PNA-FISH performance due to sample type. In

Table 1 Time to Identification of isolates from blood and peritoneal fluids by culture and PNA-FISH

\begin{tabular}{|c|c|c|c|c|c|}
\hline \multicolumn{6}{|c|}{ BLOOD CULTURES } \\
\hline Organism & $\mathbf{N}$ & $\begin{array}{c}\text { Mean Culture } \\
\text { Hours to ID (range) }\end{array}$ & $\begin{array}{l}\text { Mean PNA-FISH } \\
\text { Hours to ID (range) }\end{array}$ & $\begin{array}{c}\text { Mean Hours Saved } \\
\text { with PNA-FISH (range) }\end{array}$ & $P$ (Time Saved) \\
\hline \multirow[t]{2}{*}{ All Gram Positive } & 54 & $78.5 \mathrm{~h}$ & $8.3 \mathrm{~h}$ & $70.2 \mathrm{~h}$ & $<0.0001$ \\
\hline & & $(20-112)$ & $(3-22)$ & $(1-94)$ & \\
\hline \multirow[t]{2}{*}{ CoNS } & 34 & 72.2 & $12.5 \mathrm{~h}$ & $59.7 \mathrm{~h}$ & $<0.0001$ \\
\hline & & $(20-103)$ & $(3-22)$ & $(1-93)$ & \\
\hline \multirow[t]{2}{*}{ S. aureus } & 10 & $79.0 \mathrm{~h}$ & $9.9 \mathrm{~h}$ & $65.8 \mathrm{~h}$ & $<0.0001$ \\
\hline & & $(47-98)$ & $(3-20)$ & $(33-93)$ & \\
\hline \multirow[t]{2}{*}{ E. faecalis } & 5 & $73.8 \mathrm{~h}$ & $5.8 \mathrm{~h}$ & $68.0 \mathrm{~h}$ & $<0.0001$ \\
\hline & & $(60-97)$ & $(3-15)$ & $(45-94)$ & \\
\hline \multirow{2}{*}{$\begin{array}{l}\text { Other Enterococci } \\
\text { (includes E. faecium) }\end{array}$} & 5 & $89.0 \mathrm{~h}$ & $6.4 \mathrm{~h}$ & $82.6 \mathrm{~h}$ & $<0.0001$ \\
\hline & & $(70-112)$ & $(3-20)$ & $(69-93)$ & \\
\hline \multirow[t]{2}{*}{ All Gram Negative } & 19 & $76.2 \mathrm{~h}$ & $12.1 \mathrm{~h}$ & $64.1 \mathrm{~h}$ & 0.0002 \\
\hline & & $(23-112)$ & $(3-24)$ & $(3-115)$ & \\
\hline \multirow[t]{2}{*}{ E. coli } & 9 & $78.4 \mathrm{~h}$ & $9.2 \mathrm{~h}$ & $69.4 \mathrm{~h}$ & $<0.0001$ \\
\hline & & $(25-108)$ & $(5-18)$ & $(20-91)$ & \\
\hline \multirow[t]{2}{*}{ K. pneumoniae } & 5 & $71.6 \mathrm{~h}$ & $17.8 \mathrm{~h}$ & $54.0 \mathrm{~h}$ & 0.0109 \\
\hline & & $(23-112)$ & $(5-24)$ & $(3-91)$ & \\
\hline \multirow[t]{2}{*}{ P. aeruginosa } & 5 & $70.0 \mathrm{~h}$ & $7.8 \mathrm{~h}$ & $58.8 \mathrm{~h}$ & 0.0002 \\
\hline & & $(40-94)$ & $(3-22)$ & $(18-90)$ & \\
\hline \multirow[t]{2}{*}{ All Candida } & 4 & $96.0 \mathrm{~h}$ & $13.2 \mathrm{~h}$ & $83.0 \mathrm{~h}$ & 0.0095 \\
\hline & & $(72-130)$ & $(7.2-17.2)$ & $(65-115)$ & \\
\hline C. albicans & $2^{*}$ & $72.0 \mathrm{~h}$ & $7.2 \mathrm{~h}$ & $64.8 \mathrm{~h}$ & N/A \\
\hline C. glabrata & 1 & $130 \mathrm{~h}$ & $15.2 \mathrm{~h}$ & $114.8 \mathrm{~h}$ & N/A \\
\hline C. parapsilosis & 1 & 86 & $17.2 \mathrm{~h}$ & $68.8 \mathrm{~h}$ & NA \\
\hline \multicolumn{6}{|c|}{ PERITONEAL FLUID CULTURES ${ }^{\dagger}$} \\
\hline \multirow[t]{2}{*}{ All Gram Positive } & 5 & $73.2 \mathrm{~h}$ & $10.6 \mathrm{~h}$ & $62.6 \mathrm{~h}$ & 0.0070 \\
\hline & & $(23-111)$ & $(2-22.2)$ & $(21-90.8)$ & \\
\hline CoNS & 1 & $111.0 \mathrm{~h}$ & $19.6 \mathrm{~h}$ & $91.4 \mathrm{~h}$ & N/A \\
\hline \multirow[t]{2}{*}{ E. faecalis } & 2 & $35.0 \mathrm{~h}$ & $4.5 \mathrm{~h}$ & 30.5 & $0.1306^{\ddagger}$ \\
\hline & & $(23-47)$ & $(2-7)$ & $(21-40)$ & \\
\hline \multirow{2}{*}{$\begin{array}{l}\text { Other Enterococci } \\
\text { (includes E. faecium) }\end{array}$} & 2 & $92.5 \mathrm{~h}$ & $12.1 \mathrm{~h}$ & $80 . h$ & $0.0522^{\ddagger}$ \\
\hline & & $(75-110)$ & $(5-20)$ & $(70-90)$ & \\
\hline All Gram Negative & 2 & $101.5 \mathrm{~h}$ & $22.2 \mathrm{~h}$ & $79.3 \mathrm{~h}$ & 0.0204 \\
\hline \multirow[t]{2}{*}{ K. pneumoniae } & 2 & $101.5 \mathrm{~h}$ & $22.2 \mathrm{~h}$ & $79.3 \mathrm{~h}$ & 0.0204 \\
\hline & & $(90-113)$ & $(22.2)$ & $(67.8-90.8)$ & \\
\hline
\end{tabular}

* One isolate not identified by PNA-FISH.

+ PNA-FISH is not FDA approved for this sample type.

${ }^{\ddagger}$ Not statistically significant. 
peritoneal fluid cultures $(\mathrm{n}=17), 11$ specimens signaled positive for bacterial growth. Among signal-positive blood culture bottles containing Gram positive bacteria, the following species were identified: $2 \mathrm{E}$. faecalis, $2 \mathrm{E}$. faecium, 1 CoNS. Four bottles contained Streptococcus viridans, and subsequently could not be identified by PNA-FISH. Two signal-positive bottles contained Gram negative bacteria; $2 / 2$ isolates were identified as $K$. pneumoniae by PNA-FISH. During the period of the study, no peritoneal infections with Candida sp. were noted among clinical specimens submitted to the laboratory. Among the 6 peritoneal fluid bottles spiked with Candida sp. (3 C. albicans, 2 C. glabrata, 1 C. tropicalis) all species were correctly identified by PNA-FISH [Figure 2]. Results from spiked bottles were included only in the accuracy calculation; comparative time to detection was not calculated. Gram positive organisms were identified 62.6 hours faster with PNA-FISH compared to culture (95\% CI 2.8 to $132.1, \mathrm{p}=0.0070$ ) and Gram negative organisms were identified 79.3 hours faster with PNAFISH (95\% CI 29.82 to $128.78, \mathrm{p}=0.0204$ ) [Table 1]. Overall mean time saved using PNA-FISH for detection of Gram positive and Gram negative organisms in peritoneal fluid cultures was 70.95 hours (95\% CI 5.2 to 136.7, $\mathrm{p}=0.0435$ ). The overall accuracy for detection of bacteria and Candida sp. in peritoneal fluid was $100 \%$ (6/6, 95\% CI $54 \%$ to $100 \%)$. During testing of blood and peritoneal fluids, all PNA-FISH control slides performed as expected, thus the inter-run precision of the test was $100 \%$.

\section{Projected pharmaceutical costs}

Antifungal/antimicrobial costs were extrapolated based on organism identification from blood, time to identification, and chart review of therapy [Table 2]. In 2 of 3 patients in which C-ID, F-ID, and chart review was available, two patients were empirically treated with caspofungin for suspected C. glabrata fungemia. When identification was finalized by conventional methods as $C$. albicans and C. parapsilosis, therapy was changed to fluconazole. Using PNA-FISH, identification of C. albicans and $C$. parapsilosis could have been achieved in an average of $1 / 2$ day, compared to 4 days with $C$-ID, resulting in potential antifungal cost savings due to change of therapy from caspofungin to fluconazole of $\$ 377.74$ per day.

Four patients were selected for chart review in order to determine the potential effect of time to culture result on antibiotic costs. Each case tested positive for CoNS in blood cultures by C-ID and F-ID. Upon initial positive blood culture, patients were treated with vancomycin until culture identification of CoNS by C-ID was verified. At that point, based on clinical presentation, CoNS was considered a contaminant, resulting in discontinuation of vancomycin therapy. The time to result by C-ID was compared to the time to result by F-ID, and potential time to result saved by F-ID calculated. In this small subset of patients, the use of PNA-FISH, for rapid identification of CoNS, could have resulted in discontinuation of IV vancomycin an average of 2.2 days earlier, with an estimated savings of $\$ 20.00 /$ day [Table 2].

\section{Discussion}

PNA-FISH has been shown to be a useful and accurate method in the identification of Gram positive, Gram negative, and Candida sp. from blood cultures and peritoneal fluid cultures. When analyzing PNA-FISH probe performance for both bacteria and yeast combined in both sample types, we demonstrated an overall identification accuracy of 98.9\% (96/97). Accuracy of PNA-FISH for bacteria was $100 \%$ (80/80), accuracy for Candida sp. was $94.1 \%$ (16/17). This high level of accuracy with PNAFISH has been replicated in other studies [6-10,12,15]. We

Table 2 Potential pharmaceutical cost savings with PNA-FISH

\begin{tabular}{|c|c|c|c|c|c|c|c|}
\hline Case & $\begin{array}{c}\text { Final } \\
\text { Species } \\
\text { ID }\end{array}$ & Initial Rx & $\begin{array}{l}\text { Rx initiation until } \\
\text { change (days) }\end{array}$ & $\begin{array}{l}\text { Time to culture } \\
\text { ID (days) }\end{array}$ & $\begin{array}{l}\text { Time to PNA- } \\
\text { FISH ID (days) }\end{array}$ & $\begin{array}{l}\text { Potential time saved w/ } \\
\text { PNA-FISH (days) }\end{array}$ & $\begin{array}{c}\text { Potential cost saved } \\
\text { w/PNA-FISH }\end{array}$ \\
\hline 1 & C. albicans & Caspofungin & 6 & 3 & 0.3 & 2.7 & $\$ 1,093.50$ \\
\hline 2 & C. glabrata & Fluconazole & 2 & 5.4 & 0.6 & 4.8 & $\begin{array}{c}\text { Fluconazole changed } \\
\text { to Caspofungin }\end{array}$ \\
\hline 3 & $\frac{\text { C. }}{\text { parapsilosis }}$ & Caspofungin & 5 & 3.6 & 0.7 & 2.9 & $\$ 1,174.50$ \\
\hline 1 & CONS & Vancomycin & 3 & 3.7 & 0.8 & 2.9 & $\$ 58.00$ \\
\hline 2 & CoNS & Vancomycin & 4 & 2.1 & 0.2 & 1.9 & $\$ 38.00$ \\
\hline 3 & CoNS & Vancomycin & 5 & 3.2 & 0.3 & 2.9 & $\$ 58.00$ \\
\hline 4 & CoNS & Vancomycin & 2 & 0.8 & 0.7 & 0.1 & $\$ 2.00$ \\
\hline
\end{tabular}

${ }^{\dagger}$ Potential time saved $\mathrm{w} / \mathrm{PNA}-\mathrm{FISH} \mathrm{X}$ AWP $=$ Potential cost saved $\mathrm{w} / \mathrm{PNA}-\mathrm{FISH}$. AWP:

Fluconazole $400 \mathrm{mg}$ po/day $=\$ 27.26$ Caspofungin 50mg IV/day $=\$ 405.00$

Vancomycin $2 \mathrm{~g} / \mathrm{day}=\$ 20.00$. 
did not test identification accuracy in BACTEC-negative bottles, as PNA-FISH requires an organism concentration of at least $10^{5} \mathrm{CFU} / \mathrm{mL}$ for detection.

Clinical isolates identified in this study are representative of the most commonly reported pathogens implicated in nosocomial bloodstream infections in the United States [2]. In blood cultures, accuracy of PNAFISH for bacteria was $100 \%$. In this study, accuracy for yeast identification was $90.9 \%$ (10/11). A single weakly reactive $C$. albicans isolate may have been due to a mismatch between organism rRNA and PNA-FISH probe secondary to point mutations in sequence, as have been described by other authors [11,12].

Mean time saved using PNA-FISH for blood cultures compared to traditional culture methods averaged 72.4 hours from the time the organism (bacteria or yeast) was detected by Gram stain from a positive blood culture bottle until final identification of species [Table 1]. The significant difference in time of C-ID as compared to F-ID may partially be attributed to conventional laboratory workflow with C-ID; after subculture of a positive blood culture bottle, plate cultures were assessed once daily for adequate growth. Panels used for automated bacterial identification were set up once daily if plate culture growth was sufficient followed by 24-hour incubation. An advantage of the PNA-FISH methodology is ease of use, in that multiple final identifications may be generated in a single laboratory shift directly from positive blood culture bottles, without need for additional incubation.

The use of blood culture media is a useful method of enhancing recovery of microorganisms from peritoneal fluids [16-18]. Although not specifically FDA-approved for this indication, we also assessed performance of PNA-FISH using peritoneal fluid specimens incubated in blood culture bottles, as proof of concept. To the best of our knowledge this study is the first application of this PNA-FISH method for this sample type. Identification accuracy for both bacteria and Candida sp. was $100 \%$. Although mean time saved with PNA-FISH was not significant for Enterococcus sp., this was likely attributable to low sample numbers (Table 1).

Laboratory-spiked bottles were not used for turnaround time determination for Candida sp. in peritoneal fluids, as the intent of the study was to mimic actual clinical performance of PNA-FISH from blood culture media as much as possible. Although the turnaround time for Candida sp. could not be specifically determined due to the use of spiked bottles, based on blood culture data, it is reasonable to assume a similar reduction in time to F-ID in peritoneal fluid samples would also apply. Low numbers of peritoneal fluids tested is a limitation of this study. Although blood culture bottles containing peritoneal fluid spiked with Candida sp. were used to increase numbers to statistically meaningful levels, every attempt was made to apply PNA-FISH to situations found in actual clinical practice. Excellent performance of PNA-FISH with this sample type is encouraging for application to larger scale studies required to truly assess assay performance.

Accurate identification of pathogens is a primary driver of antimicrobial or antifungal selection. Delays in appropriate therapy clearly affect patient outcomes in a negative fashion. Among 492 intensive care patients studied by Ibrahim et al., 30\% received inadequate antimicrobial therapy for bacteremia; hospital mortality rate was $62 \%$ compared to $28.4 \%$ of patients receiving appropriate antibiotics [3]. In terms of yeast identification, both SENTRY and EIEIO sentinel surveillance programs demonstrated a shift in pathogenic Candida species over the last decade [19]. These findings support the importance of rapid identification of yeast to species level as critical to direct appropriate antifungal therapy. Excellent performance by PNA-FISH in both bacteria and yeast will address these clinical needs.

In this study, based on a limited chart review of clinical cases, the more expensive antifungal caspofungin was used empirically for 5-6 days in 2 cases in which the organism was C. albicans or C. parapsilosis fully susceptible to fluconazole. Based on our estimated antifungal cost savings, and 2009 AWP data, this could potentially result in pharmaceutical cost savings of $\$ 1,888.70$ over 5 days of therapy. Significant pharmaceutical cost savings due to de-escalation of therapy from an echinocandin to fluconazole in infections caused by fluconazole -susceptible C. glabrata have also been noted in other studies [20].

We also examined the costs associated with the use of vancomycin for CoNS infection. In four cases in which chart review was available, empiric therapy with vancomycin was continued for 2-5 days and not discontinued until CoNS was identified and deemed a contaminant, based on clinical presentation of the patient. Avoiding unnecessary use of vancomycin in these instances could save an estimated $\$ 20.00$ daily per patient. Based on our study, and use of PNA-FISH, more appropriate use of vancomycin and caspofungin could reduce hospital pharmaceutical costs. As indicated in other studies, rapid organism identification and judicious use of antibiotics has overall broader implications in antibiotic stewardship and may positively affect reduction of antibiotic resistance and patient mortality $[6,8,21]$.

Based upon chart review, a disparity was noted between time of C-ID or F-ID, and the time of therapy initiation until a change in therapy was made based on ID results (Table 2). We cannot specifically comment why, in some cases, antifungal or antibiotic therapy was not changed after organism ID was finalized. This obviously affects overall estimates of pharmaceutical cost. Based 
on traditional practice patterns, it can only be assumed that if patients are improving under a given course of therapy, clinicians are less likely to change therapy, even with the availability of ID results. Proactive management of identification results by stakeholders such as Infectious Disease or Pharmacy, as part of an active antibiotic stewardship program will contribute to the downstream benefits of rapid diagnosis by PNA-FISH.

A limitation of PNA-FISH is a requirement of an organism concentration of at least $10^{5} \mathrm{CFU} / \mathrm{mL}$ for detection. This requirement may prove to be problematic for detection of slow-growing, or fastidious organisms. At the time this study was performed, a limited number of PNA-FISH probes were available. However, additional specific PNA-FISH probes are now available for Group B Streptococcus, GNR Traffic Light (FDA-approved), C. dubliniensis, C. parapsilosis, K. pneumoniae, and Acinetobacter (analyte-specific reagents). Recent FDA approval of a more rapid PNA-FISH protocol should prove to be advantageous in further decreasing turnaround time to organism identification [9].

\section{Conclusion}

This study expands the body of knowledge evaluating PNA-FISH, a useful method for identification of organisms directly from blood culture bottles. Our study demonstrated excellent accuracy of identification of organisms for which PNA-FISH probes were available. This includes the most commonly isolated species implicated in bacteremia and fungemia. In addition, successful use of PNA-FISH in peritoneal fluid cultures could be extremely beneficial in analysis of this sample type. Turnaround time for final identification from both blood cultures and peritoneal fluid was significantly reduced in comparison to traditional culture methods. A recently approved shortened hybridization protocol will further contribute to a reduction in laboratory turnaround time. Although the correct choice of a PNA-FISH probe is contingent upon correct interpretation of a Gram stain, we do not feel this is an issue with properly trained laboratory technologists. PNA-FISH is easy to perform in the clinical laboratory and does not require significant capital equipment costs unlike microarrays or MALDITOF. PNA-FISH requires a microscope equipped with a fluorescent lamp and dual band filters for interpretation of results. Of primary importance, the accuracy and specificity of PNA-FISH can significantly affect antibiotic and antifungal utilization, allowing for more targeted therapy, reduction of duration of therapy, with an overall reduction of healthcare costs, and increased benefit to patients.

\section{Abbreviations}

PNA-FISH: Peptide nucleic acid fluorescent in situ hybridization; BSI: Bloodstream associated infections; MALDI-TOF: Matrix-assisted laser desorption ionization time of flight mass spectrometry; CoNS: Coagulasenegative Staphylococcus; C-ID: Time to identify with culture; F-ID: Time to identify with PNA-FISH; AWP: Average wholesale price.

\section{Competing interests}

The authors declare that they have no competing interests.

\section{Authors' contributions}

DMH performed laboratory testing, data acquisition, chart review and pharmaceutical cost analysis. DJH contributed to study design, data analysis, and result interpretation. All authors contributed to the preparation of the manuscript. All authors have read and approved the final manuscript.

\section{Previous presentations}

This study was presented in part at the $49^{\text {th }}$ Interscience Conference on Antimicrobial Agents and Chemotherapy, San Francisco, CA, Sept. 12 - 15, 2009

\section{Acknowledgements}

We would like to acknowledge Aaron Odegard, the Clinical Microbiology Laboratory at Mayo Clinic in Jacksonville, FL, and the Mycology Laboratory at Mayo Clinic in Rochester, MN for technical assistance. We would also like to acknowledge BioMérieux, Inc. and AdvanDx Inc. for supplying testing equipment and test kits.

\section{Author details}

${ }^{1}$ Division of Hospital Internal Medicine, Mayo Clinic College of Medicine, 4500 San Pablo Rd, Jacksonville, Florida 32224, USA. ²Department of Laboratory Medicine and Pathology, Mayo Clinic College of Medicine, 4500 San Pablo Rd, Jacksonville, Florida 32224, USA.

Received: 13 December 2012 Accepted: 5 January 2013

Published: 7 January 2013

\section{References}

1. Heron M, Hoyert DL, Murphy SL, Xu J, Kochanek KD, Tejada-Vera B: Deaths: final data for 2006. Nat/ Vital Stat Rep 2009, 57(14):1-134

2. Wisplinghoff $H$, Bischoff $T$, Tallent SM, Seifert $H$, Wenzel RP, Edmond MB: Nosocomial bloodstream infections in US hospitals: analysis of 24,179 cases from a prospective nationwide surveillance study. Clin Inf Dis 2004, 39(3):309-317.

3. Ibrahim EH, Sherman G, Ward S, Fraser VJ, Kollef MH: The influence of inadequate antimicrobial treatment of bloodstream infections on patient outcomes in the ICU setting. Chest 2000, 118(1):146-155.

4. Baron EJ, Weinstein MP, Dunne WMJ, Yagupsky P, Welch DF, Wilson DM: Cumitech 1C: Blood Cultures N. 1st edition. Washington D.C: ASM Press; 2005.

5. Klouche M, Schroder U: Rapid methods for diagnosis of bloodstream infections. Clin Chem Lab Med 2008, 46(7):888-908.

6. Forrest GN, Roghmann MC, Toombs LS, Johnson JK, Weekes E, Lincalis DP, Venezia RA: Peptide nucleic acid fluorescent in situ hybridization for hospital-acquired enterococcal bacteremia: delivering earlier effective antimicrobial therapy. Antimicrob Agents Chemother 2008, 52(10):3558-3563.

7. Sogaard M, Hansen DS, Fiandaca MJ, Stender H, Schonheyder HC: Peptide nucleic acid fluorescence in situ hybridization for rapid detection of Klebsiella pneumoniae from positive blood cultures. J Med Microbiol 2007, 56(Pt 7):914-917.

8. Forrest GN, Mehta S, Weekes E, Lincalis DP, Johnson JK, Venezia RA: Impact of rapid in situ hybridization testing on coagulase-negative staphylococci positive blood cultures. J Antimicrob Chemother 2006, 58(1):154-158.

9. Morgan M, Marlowe E, Della-Latta P, Salimnia H, Novak-Weekley S, Wu F, Crystal BS: Multicenter evaluation of a new shortened peptide nucleic acid fluorescence in situ hybridization procedure for species identification of select Gram-negative bacilli from blood cultures. J Clin Microbiol 2010, 48(6):2268-2270.

10. Reller ME, Mallonee AB, Kwiatkowski NP, Merz WG: Use of peptide nucleic acid-fluorescence in situ hybridization for definitive, rapid identification of five common Candida species. J Clin Microbiol 2007, 45(11):3802-3803.

11. Rigby S, Procop GW, Haase G, Wilson D, Hall G, Kurtzman C, Oliveira K, Von Oy S, Hyldig-Nielsen JJ, Coull J, et al: Fluorescence in situ hybridization 
with peptide nucleic acid probes for rapid identification of Candida albicans directly from blood culture bottles. J Clin Microbiol 2002, 40(6):2182-2186

12. Shepard JR, Addison RM, Alexander BD, Della-Latta P, Gherna M, Haase G, Hall G, Johnson JK, Merz WG, Peltroche-Llacsahuanga H, et al: Multicenter evaluation of the Candida albicans/Candida glabrata peptide nucleic acid fluorescent in situ hybridization method for simultaneous dualcolor identification of C. albicans and C. glabrata directly from blood culture bottles. J Clin Microbiol 2008, 46(1):50-55.

13. GraphPad Software QuickCalcs. http://graphpad.com/quickcalcs.

14. Book R: Pharmacy Fundamental Reference. 113th edition. Montvale, New Jersey: Physicians Desk Reference Inc; 2009.

15. Hall L, Le Febre KM, Deml SM, Wohlfiel SL, Wengenack NL: Evaluation of the Yeast Traffic Light PNA FISH probes for identification of Candida species from positive blood cultures. I Clin Microbiol 2012 50(4):1446-1448.

16. Akcam FZ, Yayli G, Uskun E, Kaya O, Demir C: Evaluation of the Bactec microbial detection system for culturing miscellaneous sterile body fluids. Res Microbiol 2006, 157(5):433-436.

17. Fuller DD, Davis TE: Comparison of BACTEC plus Aerobic/F, Anaerobic/F, Peds Plus/F, and Lytic/F media with and without fastidious organism supplement to conventional methods for culture of sterile body fluids. Diagn Microbiol Infect Dis 1997, 29(4):219-225.

18. Sorlin P, Mansoor I, Dagyaran C, Struelens MJ: Comparison of resincontaining BACTEC Plus Aerobic/F* medium with conventional methods for culture of normally sterile body fluids. J Med Microbiol 2000, 49(9):787-791.

19. Pfaller MA, Diekema DJ: Role of sentinel surveillance of candidemia: trends in species distribution and antifungal susceptibility. J Clin Microbiol 2002, 40(10):3551-3557.

20. Collins CD, Eschenauer GA, Salo SL, Newton DW: To test or not to test: a cost minimization analysis of susceptibility testing for patients with documented Candida glabrata fungemias. J Clin Microbio/ 2007, 45(6):1884-1888.

21. Hermsen ED, Shull SS, Klepser DG, Iwen PC, Armbrust A, Garrett J, Freifeld AG, Rupp ME: Pharmacoeconomic analysis of microbiologic techniques for differentiating staphylococci directly from blood culture bottles. J Clin Microbiol 2008, 46(9):2924-2929.

doi:10.1186/1476-0711-12-2

Cite this article as: Harris and Hata: Rapid identification of bacteria and candida using pna-fish from blood and peritoneal fluid cultures: a retrospective clinical study. Annals of Clinical Microbiology and Antimicrobials 2013 12:2.

\section{Submit your next manuscript to BioMed Central and take full advantage of:}

- Convenient online submission

- Thorough peer review

- No space constraints or color figure charges

- Immediate publication on acceptance

- Inclusion in PubMed, CAS, Scopus and Google Scholar

- Research which is freely available for redistribution

Submit your manuscript at www.biomedcentral.com/submit
Biomed Central 